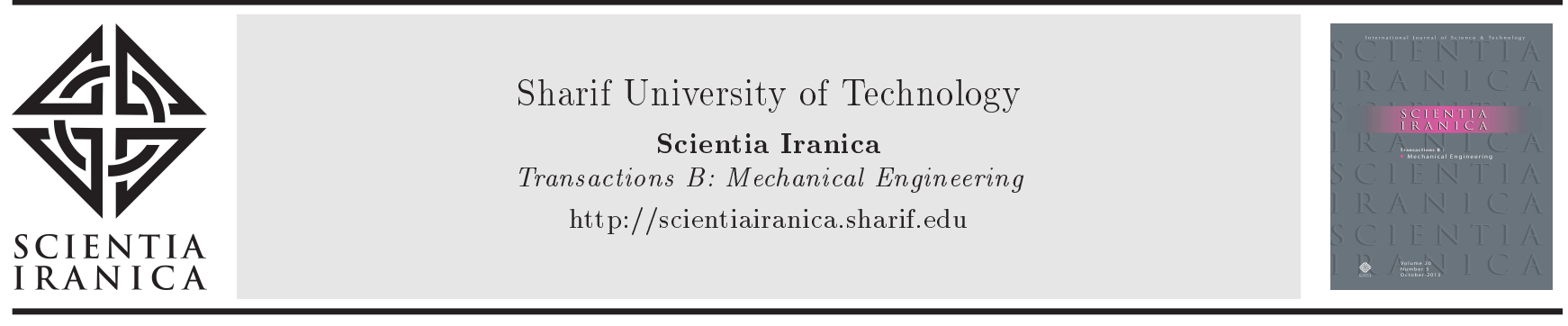

\title{
Optimization of manipulator drive mechanisms of hydraulic excavators on the basis of the tribological criterion
}

\author{
J. Pavlović*, D. Janošević, and V. Jovanović \\ Faculty of Mechanical Engineering, University of Nǐs, A. Medvedeva 14, 18000 Niš, Serbia.
}

Received 13 March 2018; received in revised form 1 April 2019; accepted 20 May 2019

\section{KEYWORDS \\ Hydraulic excavators; \\ Drive mechanisms; \\ Optimization; \\ Tribological criterion; \\ Multibody dynamics.}

\begin{abstract}
The paper analyzes functional, structural, and tribological parameters of kinematic pairs (joints) of the kinematic chain and manipulator drive mechanisms of hydraulic excavators. To carry out the related analysis, the tribological criterion ensures the optimal synthesis of manipulator drive mechanisms of hydraulic excavators. This criterion determines the mechanical efficiency of drive mechanisms to reflect the tribological loss of the power of the excavator driving mechanism due to the friction between elements of the joints in manipulator drive mechanisms. This paper provides experimental results of tribological research and tribological criterion during the examination and synthesizes manipulator drive mechanisms of a hydraulic excavator with $17,000 \mathrm{~kg}$ in mass.

(C) 2020 Sharif University of Technology. All rights reserved.
\end{abstract}

\section{Introduction}

Hydraulic excavators, of all sizes, are characterized by a large number of manipulators that basically consist of kinematic chains with linkage elements connected by rotating kinematic pairs, i.e., fifth-class joints. Manipulator drive mechanisms comprise manipulator kinematic pairs linked directly or indirectly to two-way hydraulic cylinders.

Existing studies on the optimization of manipulator drive mechanisms have considered the following optimization criteria: minimum stress in the excavators' kinematic chain link $[1,2]$, maximum transferable function of the manipulator drive mechanisms [3,4], maximum working area of the manipulator [5], and maximum dynamic stability [6]. Besides, recently, the

*. Corresponding author. Tel.: +38118500679

E-mail addresses: jovan.pavlovic@masfak.ni.ac.rs (J.

Pavlović); dragoslav.janosevic@masfak.ni.ac.rs (D.

Janosević); vesna.jovanovic@masfak.ni.ac.rs (V. Jovanović)

doi: $10.24200 /$ sci.2019.50617.1790 optimal synthesis of the slewing platform drive mechanisms of hydraulic excavators has received significant attention [7].

During the manipulation task of hydraulic excavators, the elements of manipulator kinematic pairs experience a pronounced relative movement under the loading accompanied by tribological phenomena, i.e., friction and wear, between the elements of the machine's kinematic chain joints. The consequences of tribological phenomena are loss of energy of drive mechanisms and the reduction of the lifecycle of joint elements $[8,9]$.

This paper first analyzes the functional, structural, and tribological parameters of kinematic chain joints in the excavating manipulator of a hydraulic excavator and, then, defines the tribological criterion for the optimal determination of parameters of manipulator drive mechanisms on the basis of the loss of energy due to friction in mechanism joints.

\section{Mathematical model}

To analyze tribological parameters and define the 


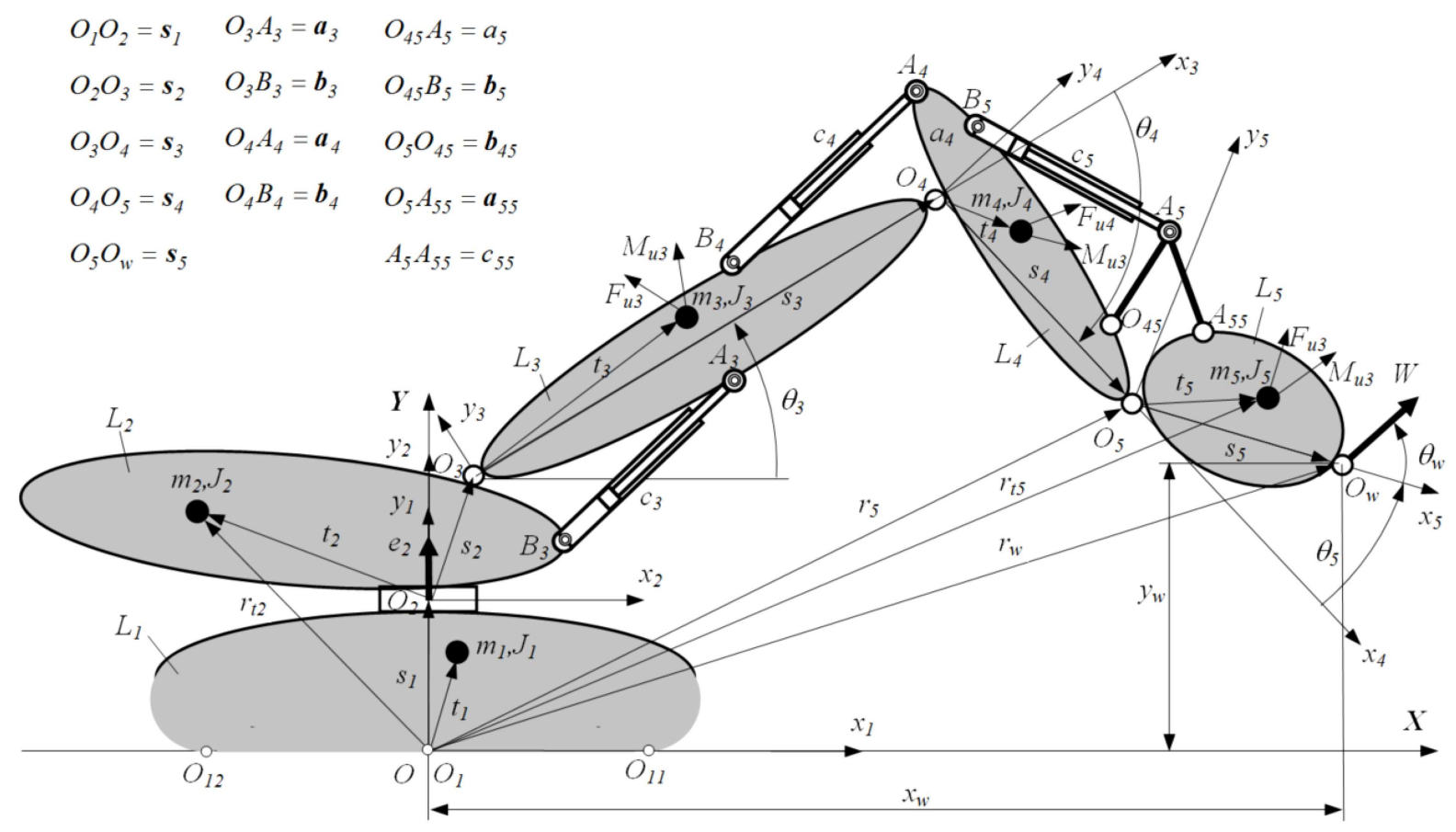

Figure 1. Schematic representation of the excavator's kinematic chain.

tribological optimization criterion of manipulator drive mechanisms of a hydraulic excavator, a mathematical model composed of a mathematical model of the kinematic chain and a mathematical model of the manipulator drive mechanisms was developed.

The mathematical model of the kinematic chain encompasses the general five-link configuration of the excavator that comprises the support and movement link $L_{1}$ (Figure 1), the rotation link (platform) $L_{2}$, and the three-link excavating manipulator with the boom $L_{3}$, stick $L_{4}$, and bucket $L_{5}$. The links of the adopted kinematic chain of the excavator form fifthclass kinematic pairs and rotary joints with a single degree of freedom.

The support and movement link of the excavator and the support surface form a third-class zero joint with potential movements on the plane of the surface. The support and movement link and the rotation link (platform) form a kinematic pair connected to the fifthclass rotary joint $\mathrm{O}_{2}$ with elements in the form of rolling axial bearing. The center of joint $\mathrm{O}_{2}$ is the point of the perpendicular intersection of the axis of joints through the plane that contains the centers of rolling elements of axial bearings. The manipulator kinematic chain is of planar configuration. Centers of the manipulator joints $O_{i}(i=3,4,5)$ are the intersection points of the axis of joints and the plane of symmetry of the manipulator chain links. The intersection of the bucket cutting edge and the plane of the manipulator represents the center of the bucket cutting edge $O_{w}$.

The assumptions about the mathematical model of the excavator kinematic chain are as follows:
(1) The support surface and excavator kinematic chain links are modeled using rigid bodies;

(2) The first joint between the support and movement link and the excavator support surface is characterized by a variable position and form, thus acting as a translatory-sliding joint along the contact between the support and movement link and the surface while having the form of rotary joints $O_{11}$, and $O_{12}$, whose axes represent potential (longitudinal $\mathrm{x}-\mathrm{x}$ or transverse $\mathrm{z}-\mathrm{z}$ ) (Figure 1) excavator tip-over lines on the edges of the contact;

(3) During the manipulation task, the excavator is subjected to external (technological) forces including digging resistive force $W$, internal and gravitational forces of kinematic chain links, driving mechanism links, and material excavated with the excavator bucket;

(4) During the digging operation, the kinematic chain of the excavator is observed as an open configuration chain, whose final link, the bucket, is subjected to digging resistive force $W$ at the centre of the cutting edge and on the plane of the manipulator.

The area of the excavator model is determined by an absolute coordinate system $O X Y Z$ (Figure 1) with unit vectors $i, j, k$ along the coordinate axes. The excavator support surface lies on the $O X Z$ horizontal plane of the absolute coordinate system, while the $O Y$ vertical axis of the same system falls on the axis of the 
support link, i.e., rotational kinematic pair, when the excavator is positioned on the horizontal surface.

A link of the kinematic chain, $L_{i}$, in its local coordinate system $O_{i} x_{i} y_{i} z_{i}$ with unit vectors $\widehat{\mathbf{i}}_{i}, \hat{\mathbf{j}}_{i}, \widehat{\mathbf{k}}_{i}$ along the coordinate axes is defined by the following set of parameters [10]:

$$
L_{i}=\left\{\widehat{\mathbf{e}}_{i}, \widehat{\mathbf{s}}_{i}, \widehat{\mathbf{t}}_{i}, m_{i}, \widehat{\mathbf{J}}_{i}\right\},
$$

where $\widehat{\mathbf{e}}_{i}$ is the unit vector of joint $O_{i}$ axis which connects link $L_{i}$ to the previous link $L_{i-1}$ (Figure 1 ), $\widehat{\mathbf{s}}_{i}$ is the vector of the position of joint $O_{i+1}$ center used to connect the chain link $L_{i}$ to the next member $L_{i+1}$, $\widehat{\mathbf{t}}_{i}$ is the vector of the position of the member $L_{i}$ mass center, $m_{i}$ is the link mass, and $\widehat{\mathbf{J}}_{i}$ is the tensor of the moment of inertia of the link. Vector quantities marked with a 'cap' and without it are associated with the local coordinate system and the absolute coordinate system, respectively.

Transformation parameters of the drive mechanism are the parameters of hydraulic cylinders and they are determined using the mathematical model with the following set of values:

$$
C_{c i}=\left\{d_{i 1}, d_{i 2}, c_{i p}, c_{i k}, m_{c i}, n_{\mathrm{ci}}\right\} \quad \forall i=3,4,5,
$$

where $d_{i 1}$ and $d_{i 2}$ are the diameters of the piston and the connecting rod of the hydraulic cylinder; $c_{i p}$ is the minimal length of the hydraulic cylinder with a fully retracted connecting rod; $c_{i k}$ is the maximal length of the hydraulic cylinder with the fully extended connecting rod; $m_{c i}$ is the mass of the hydraulic cylinder; and $n_{c i}$ is the number of hydraulic cylinders of the drive mechanism.

Transmission parameters of the drive mechanism of the boom $C_{3}$ and stick $C_{4}$ of the manipulator in the mathematical model of the excavator are determined by the following set of values:

$$
C_{p i}=\left\{\mathbf{a}_{i}, \mathbf{b}_{i}\right\} \quad \forall i=3,4,
$$

where $\mathbf{a}_{i}$, and $\mathbf{b}_{i}$ represent the vectors of the position of joint centers in which hydraulic cylinders are connected to the links of the drive mechanism and kinematic chain.

The subset of transmission parameters of the manipulator bucket $C_{5}$ drive mechanism in the mathematical model of the excavator is determined by the following set of values:

$$
C_{p 5}=\left\{a_{5}, \mathbf{b}_{5}, c_{55}, \mathbf{a}_{55}, \mathbf{b}_{45}\right\},
$$

where $a_{5}$ is the kinematic length of the bucket hydraulic cylinder lever in the transmission part of the drive mechanism; $\mathbf{b}_{5}$ is the vector, i.e., coordinates, of the position of the joint center in which the bucket hydraulic cylinder is connected to the manipulator stick; $c_{55}$ is the kinematic length of the link of the transmission part of the drive mechanism; and $\mathbf{a}_{55}$ and $\mathbf{b}_{45}$ are the vectors, i.e., coordinates, of the position of the joint center in which the transmission levers are connected to the links of the drive mechanism kinematic pair.

The assumptions about the mathematical model of the manipulator drive mechanisms in the hydraulic excavator are as follows: (1) The position of the mass center of hydraulic cylinders is in the middle of the current length of the hydraulic cylinder; (2) The masses of joint elements belong to the links of the manipulator kinematic chain.

The internal (generalized) coordinates of the mathematical model of the excavator kinematic chain are represented by angles $\theta_{i}$ of the relative position of link $L_{i}$ in relation to the previous link $L_{i-1}$ upon rotation around the joint $O_{i}$ axis. Angles $\theta_{i} \quad(i=$ $3,4,5)$ of the relative position of the manipulator link, $L_{i}$, with respect to the previous link $L_{i-1}$ are determined based on the length $c_{i}$ of the hydraulic cylinders of the manipulator boom, stick, and bucket drive mechanisms.

Unit vector $\mathbf{e}_{i}$ of joint $O_{i}$ axis of the excavator kinematic pair in the absolute coordinate system is determined through the following equation:

$$
\mathbf{e}_{i}=\mathbf{A}_{i o} \widehat{\mathbf{e}}_{i}
$$

Unit vector $e_{1}$ of the first joint axis is directed along the potential excavator tip-over lines (Figure 1).

Vector $r_{i}$ of joint $O_{i}$ center of the excavator kinematic pair in the absolute coordinate system is determined through the following equation:

$$
\mathbf{r}_{i}=\sum_{j=1}^{i-1} \mathbf{A}_{j o} \widehat{\mathbf{s}}_{j}, \quad \forall i=2,3,4,5 .
$$

Vector $r_{w}$ of the center of the bucket cutting edge in the absolute coordinate system is determined through the following equation:

$$
\mathbf{r}_{w}=\sum_{i=1}^{5} \mathbf{A}_{i o} \widehat{\mathbf{s}}_{i} .
$$

Vector $r_{t i}$ of the center of the kinematic chain link $L_{i}$ mass in the absolute coordinate system is determined through the following equation:

$$
\mathbf{r}_{t i}=\mathbf{r}_{i}+\mathbf{A}_{i o} \widehat{\mathbf{t}}_{i}
$$

where $\mathbf{A}_{i o}$ is the homogeneous transformation matrix used to transfer the vector quantities from the local coordinate system $O_{i} x_{i} y_{i} z_{i}$ of link $L_{i}$ to the absolute coordinate system $O X Y Z$.

Kinematic quantities for the center of the chain link mass $L_{i}$ are angular velocity $\boldsymbol{\omega}_{i}$ and linear $w i$ and angular $\varepsilon_{i}$ accelerations, which are determined with respect to the absolute coordinate system using recursive equations $[11,12]$ : 


$$
\begin{aligned}
\boldsymbol{\omega}_{i}= & \boldsymbol{\omega}_{i-1}+\dot{\theta}_{\mathrm{i}} \mathrm{e}_{\mathrm{i}}, \quad \forall \mathrm{i}=1, \ldots, 5, \\
\varepsilon_{i}= & \varepsilon_{i-1}+\ddot{\theta}_{i} \mathbf{e}_{i}+\left(\boldsymbol{\omega}_{i-1} \times \dot{\theta}_{i} \mathbf{e}_{i}\right), \\
\mathbf{w}_{i}= & \mathbf{w}_{i-1}+\left(\varepsilon_{i-1} \times\left(\mathbf{s}_{i-1}-\mathbf{t}_{i-1}\right)\right)+\boldsymbol{\omega}_{i-1} \\
& \times\left(\boldsymbol{\omega}_{i-1} \times\left(\mathbf{s}_{i-1}-\mathbf{t}_{i-1}\right)\right)+\left(\varepsilon_{i} \times \mathbf{t}_{i}\right) \\
& +\left(\boldsymbol{\omega}_{i} \times\left(\boldsymbol{\omega}_{i} \times \mathbf{t}_{i}\right)\right),
\end{aligned}
$$

where $\dot{\theta}_{i}$ and $\ddot{\theta}_{i}$ are the angular velocity and angular acceleration of link $L_{i}$ in joint $O_{i}$.

\section{Tribological analysis}

Joints of the kinematic chain and drive mechanisms of the excavator manipulator form tribomechanical subsystems, whose structural parameters are shape, macro and microgeometry, the material of joint elements, and the means and manner of joint lubrication. Elements of rotary joints, fifth-class drive mechanisms of the excavator manipulator, are derived in the form of a single pair of the sliding shells 1 (Figure 2) embedded in the hub of the relatively mobile link $L_{i}$ and the clevis pin 2 linked to the relatively immobile link $L_{i-1}$ of the kinematic pair. Macrogeometry determines the basic dimensions of the joint: the diameter of the clevis pin (shaft) $d_{s i}$, the width of the sliding shell $b_{s i}$, the diameter of the hub $D_{s i}$, the span of the shells $l_{s i}$, and the span of the hub $L_{s i}$. Microgeometry relates to the quality of the surface and the type of contact between the joint elements.

Function parameters of joints in the excavator manipulator are associated with the kinematic quantities such as range $\theta_{i o}$ and angular velocity $\dot{\theta}_{i}$ of the relative movement of the links in the kinematic pair subjected to loading: force $\mathbf{F}_{r i}$ and moment $\mathbf{M}_{r i}$ of the joint elements that occur during the manipulation task - the working cycle of the excavator [13].
Force $\mathbf{F}_{r i}$ and moment $\mathbf{M}_{r i}$ of the loading of the elements of $O_{i}$ (Figure 2) are determined by using the virtual cut in the manipulator kinematic chain in the same joint and by reducing all of loads of the given part of the chain to the center of the joint $(j>i)$ :

$$
\begin{aligned}
\mathbf{F}_{r i}= & \mathbf{F}_{c i}+\mathbf{W}+\sum_{j=i}^{5} \mathbf{F}_{u j}, \quad \forall \mathrm{i}=3,4,5, \\
\mathbf{M}_{r i}= & \mathbf{M}_{c i}+\left(\mathbf{r}_{w}-\mathbf{r}_{i}\right) \times \mathbf{W}+\sum_{j=i}^{n} \mathbf{M}_{u j} \\
& +\sum_{j=i}^{n}\left(\mathbf{r}_{t j}-\mathbf{r}_{i}\right) \times \mathbf{F}_{u j}, \quad \forall \mathrm{i}=3,4,5,
\end{aligned}
$$

where $\mathbf{F}_{c i}$ is the force produced by the hydraulic cylinder of the drive mechanism, $\mathbf{M}_{c i}$ is the moment of the drive mechanism, $\mathbf{W}$ is the potential digging resistive force, $\mathbf{F}_{u j}$ is the total force in the center of the link mass, and $\mathbf{M}_{u j}$ is the moment of internal forces in the center of the link mass.

The potential digging resistive force, $\mathbf{W}$, for a certain direction of activity represents the minimal value of digging resistive force from a set of boundary values of digging resistive force which allows for the stability of the excavator and boundary resistive forces that can be overcome by manipulator drive mechanisms at the maximal pressure of the excavator hydrostatic system.

The force $F_{c i}$ and moment $M_{c i}$ quantities of drive mechanisms of the excavator manipulator are determined using the following equations:

$$
\begin{gathered}
F_{c i}=n_{c i} \cdot\left[\frac{d_{i 1}^{2} \pi}{4} p_{i 1}-\frac{\left(d_{i 1}^{2}-d_{i 2}^{2}\right) \pi}{4} p_{i 2}\right] \\
\cdot \eta_{c m i}, \quad \forall \mathrm{i}=3,4,5
\end{gathered}
$$

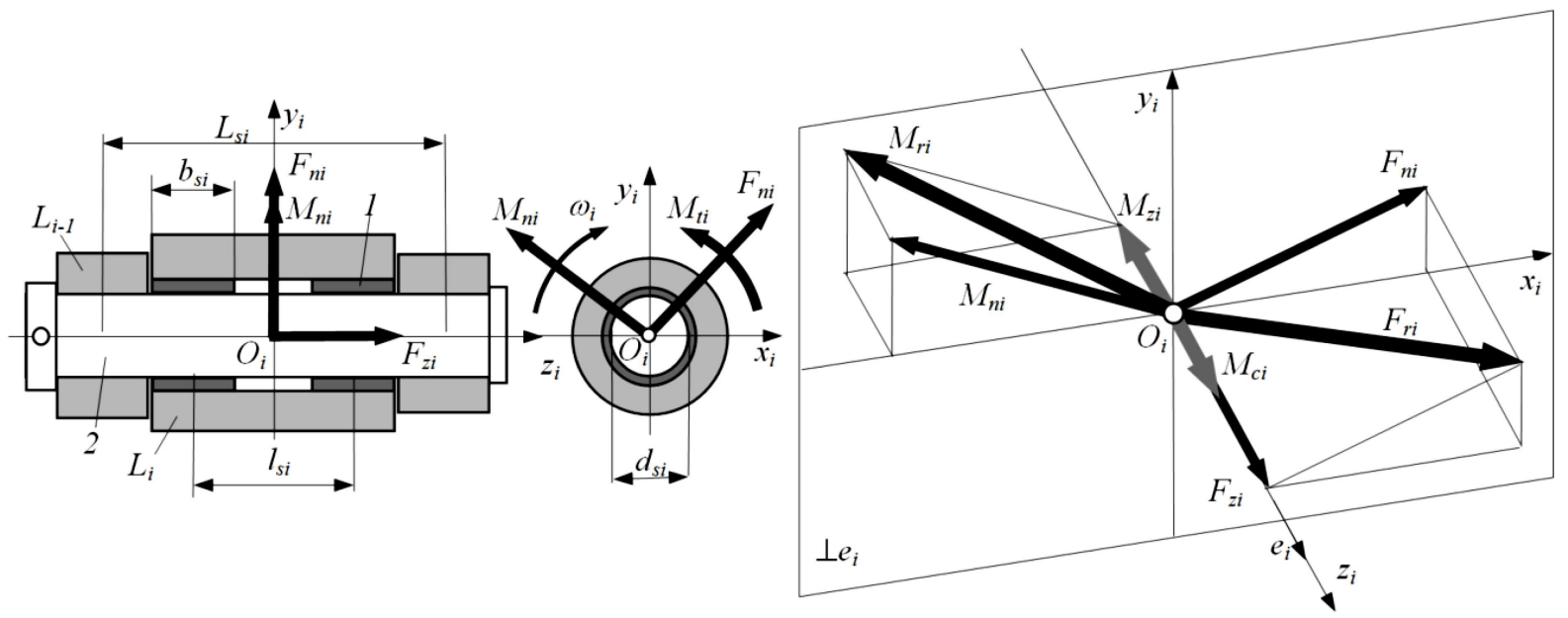

Figure 2. Loads of elements of kinematic pairs (joints) in the excavator manipulator. 
Table 1. Variant solutions of A and B excavators.

\begin{tabular}{|c|c|c|c|c|c|c|c|c|c|c|c|c|}
\hline \multirow[b]{2}{*}{$\begin{array}{c}\text { Excavator } \\
\text { variant } \\
\text { symbol }\end{array}$} & \multicolumn{4}{|c|}{ Boom mechanism } & \multicolumn{4}{|c|}{ Stick mechanism } & \multicolumn{4}{|c|}{ Bucket mechanism } \\
\hline & $\begin{array}{l}d_{31} \\
(\mathrm{~m})\end{array}$ & $\begin{array}{l}d_{32} \\
(\mathrm{~m})\end{array}$ & $\begin{array}{l}c_{3 p} \\
(\mathbf{m})\end{array}$ & $\begin{array}{l}c_{3 k} \\
(\mathbf{m})\end{array}$ & $\begin{array}{l}d_{41} \\
(\mathrm{~m})\end{array}$ & $\begin{array}{l}d_{42} \\
(\mathrm{~m})\end{array}$ & $\begin{array}{l}c_{4 p} \\
(\mathbf{m})\end{array}$ & $\begin{array}{l}c_{4 k} \\
(\mathbf{m})\end{array}$ & $\begin{array}{l}d_{51} \\
(\mathbf{m})\end{array}$ & $\begin{array}{l}d_{52} \\
(\mathrm{~m})\end{array}$ & $\begin{array}{l}c_{5 p} \\
(\mathbf{m})\end{array}$ & $\begin{array}{l}c_{5 k} \\
(\mathbf{m})\end{array}$ \\
\hline $\mathbf{A}$ & 0.115 & 0.080 & 1.533 & 2.541 & 0.140 & 0.080 & 1.738 & 2.901 & 0.115 & 0.080 & 1.358 & 2.191 \\
\hline B & 0.180 & 0.100 & 1.013 & 1.417 & 0.200 & 0.110 & 1.759 & 1.817 & 0.160 & 0.090 & 0.992 & 1.416 \\
\hline
\end{tabular}

$$
\begin{gathered}
M_{c i}=\operatorname{sign}\left(k_{i}\right) \cdot r_{c i} \cdot F_{c i}, \quad \forall i=3,4,5, \quad k_{3}=1, \\
k_{4}=k_{5}=-1
\end{gathered}
$$

where $p_{i 1}$ and $p_{i 2}$ are the pressure of the hydraulic cylinder on the piston side and on the connecting rod side of the drive mechanism, $\eta_{c m i}$ is the mechanical efficiency degree of the hydraulic cylinder, and $r_{c i}$ is the transmission function of the drive mechanism of the excavator manipulator.

The total force $\mathbf{F}_{u j}$ related to the mass center of link $L_{j}$, considering the effect of gravity, is equal to:

$$
\mathbf{F}_{u j}=m_{j} \mathbf{w}_{j}-m_{j} g \mathbf{j} .
$$

The moment of internal forces $\mathbf{M}_{u j}$ of link $L_{j}$ is determined based on Euler's dynamic equations:

$$
\begin{aligned}
& \widehat{\mathbf{M}}_{u i}=-\widehat{\mathbf{J}}_{i} \widehat{\boldsymbol{\varepsilon}}_{i}+\left(\widehat{\boldsymbol{\omega}}_{i} \times \widehat{\mathbf{J}}_{i} \widehat{\boldsymbol{\omega}}_{i}\right) \\
& \mathbf{M}_{u i}=\mathbf{A}_{i o} \widehat{\mathbf{M}}_{u i} .
\end{aligned}
$$

Loads of joint elements can be broken down into components that are collinear and perpendicular to the joint $\mathbf{e}_{\mathbf{i}}$ axis (Figure 2):

$$
\begin{aligned}
& \mathbf{F}_{r i}=\mathbf{F}_{n i}+\mathbf{F}_{z i}, \\
& \mathbf{M}_{r i}=\mathbf{M}_{n i} .
\end{aligned}
$$

During the manipulation task, the drive moment of mechanism $\mathbf{M}_{c i}$ overcomes the components of loading moments that are collinear with the joint $\mathbf{e}_{\mathbf{i}}$ axis, thus the resulting moment for the joint axis is $\mathbf{M}_{z i}=0$. The other components of joint loads $\mathbf{F}_{n i}, \mathbf{F}_{z i}, \mathbf{M}_{n i}$ stress the joint structure, while some others cause friction between its elements.

For the planar configuration of the manipulator drive mechanism, only the perpendicular force $\mathbf{F}_{n i}$ that acts on the joint depends, among other loads, on the reduced force $\mathbf{F}_{c i}$ of the hydraulic cylinder, i.e., drive mechanism parameters. The other joint loads $\mathbf{F}_{z i}, \mathbf{M}_{n i}$ are not subjected to the influence of drive mechanism parameters since the reduced force of the hydraulic cylinder acts on the plane of the manipulator perpendicular to the joint axis. The perpendicular force $\mathbf{F}_{n i}$, apart from exerting load on the joint structure, causes friction between its relatively mobile elements.

The developed software was used to perform the analysis in order to examine the effect of drive mechanism parameters on the loading of mechanism joint elements in models $\mathrm{A}$ and $\mathrm{B}$ of hydraulic excavators (Table 1) of $17,000 \mathrm{~kg}$ in mass with the identical kinematic chain of the excavating manipulator with a bucket of $0.6 \mathrm{~m}^{3}$ in volume, but with variant solutions of drive mechanisms and different transformation and transmission parameters that have the same range of movement angles $\theta_{i o}$ and the same drive moments $\mathbf{M}_{c i}$ of the executive mechanism link. In the analysis, for the variant solutions of drive mechanisms, the same parameters of the excavator hydrostatic system and the same conditions of the excavator manipulation tasks were simulated.

Some of the research results are shown graphically in a diagram (Figure $3(\mathrm{a})-(\mathrm{c})$ ) that contains the change in loading forces $\left(F_{n i}, i=3,4,5\right)$ of specific manipulator joints and a diagram (Figure $3(\mathrm{~d})$ ) that depicts the loss of power due to friction of the joints during the manipulation task of the excavator. The obtained results of the performed analysis show that variant solutions of drive mechanisms with lower transformation and higher transmission parameters possess lower loads on the joint elements and less loss of power due to friction of the joints, and vice versa. The difference between the joint load and the loss of power due to friction is particularly prominent during the digging operation when the loads on the joints are at the highest value.

Joint structure parameters depend on the drive mechanism parameters, as shown in the equation, and they are used to determine the diameter of the clevis pin (shaft) of the joints based on loads and mechanical characteristics:

$$
\begin{aligned}
& d_{s i}=\max \\
& \left\{\begin{array}{l}
\left(\frac{F_{n m i}}{2 \cdot e_{s i} \cdot p_{s m}}\right)^{1 / 2} \\
\left(\frac{2 \cdot F_{n m i}}{\pi \cdot \tau_{s m}}\right)^{1 / 2} \\
{\left[\frac{8 \cdot F_{n m i}\left(L_{s i}-l_{s i}\right)}{\pi \cdot \sigma_{s m}}\right]^{1 / 3}}
\end{array} \quad \forall i=3,4,5\right.
\end{aligned}
$$




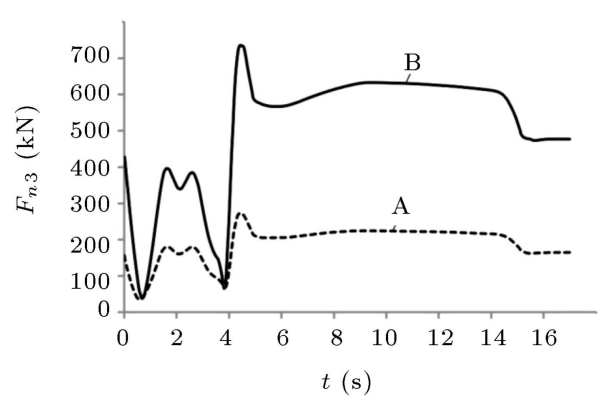

(a)

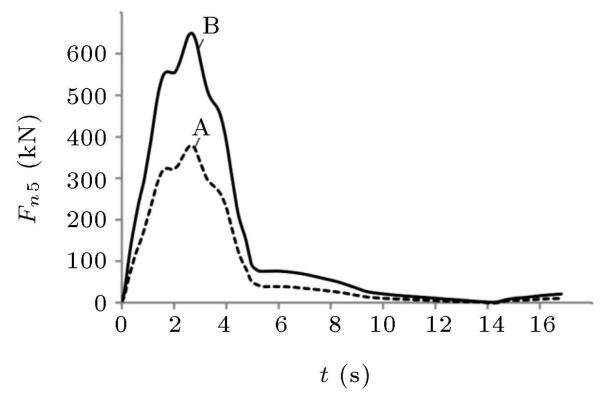

(c)

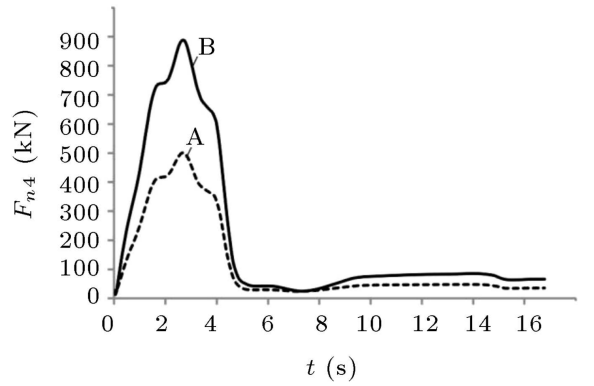

(b)

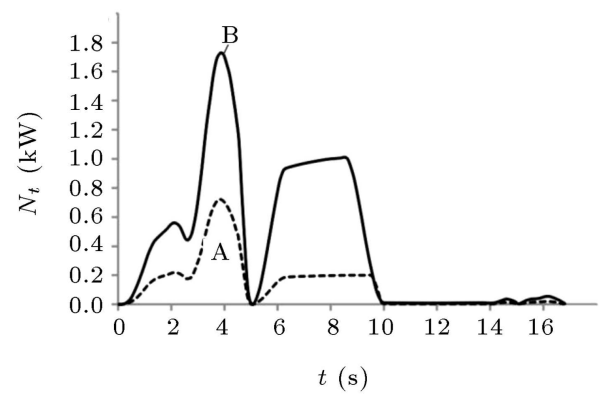

(d)

Figure 3. Tribological analysis of variant solutions of excavators A and B: (a) Forces $F_{n 3 A}$ and $F_{n 3 B}$ in joints $O_{3}$, (b) forces $F_{n 4 A}$ and $F_{n 4 B}$ in joints $O_{4}$, (c) forces $F_{n 5 A}$ and $F_{n 5 B}$ in joints $O_{5}$, and (d) losses of power $N_{t A}$ and $N_{t B}$ due to friction in manipulator joints of excavators $\mathrm{A}$ and $\mathrm{B}$.

where $F_{n m i}$ is the maximal value of the force exerted on the joint elements, acting perpendicularly on the joint axis, $e_{s i}$ is the ratio between the width of the sliding shell $l_{s i}$ and the diameter of the joint axle $d_{s i}$ (Figure 2), $p_{s m}, \tau_{s m}$, and $\sigma_{s m}$ are the allowed stress of the surface pressures, shear, and bending of the joint elements.

According to Eq. (20), for the same material of joint elements, variant solutions of drive mechanisms with lower transformation and higher transmission parameters have smaller dimensions of joint elements due to lower loads, and vice versa.

Tribological joint parameters correspond to the friction and wear between joint elements. The friction between joint elements leads to the loss of energy and power upon the transmission of energy, with thermal damage in the joint. Wear causes the loss of material and a change in the microgeometry of joint elements.

According to the parameters of joint function and structure, tribological parameters of drive mechanisms in an excavator manipulator can be singled out as follows:

a) Moment $M_{t i}$ of friction resistive force between joint $O_{i}$ elements:

$$
\mathrm{M}_{\mathrm{ti}}=-\operatorname{sign}\left(\dot{\theta}_{\mathrm{i}}\right) \frac{\mathrm{d}_{\mathrm{si}}}{2} \mu_{\mathrm{tz}} \cdot \mathrm{F}_{\mathrm{ni}}
$$

b) Power $N_{t i}$ lost due to friction resistive force between joint $O_{i}$ elements:

$$
N_{t i}=M_{t i} \cdot \dot{\theta}_{i},
$$

where $\mu_{t z}$ is the coefficient of friction between the sliding elements of a joint. Although Bojić et al. [14] in a recent study discussed the dependence of the coefficient of friction on the temperature and force acting in the joint, the assumption of the constant coefficient of friction is adopted here for further consideration. It should be noted that this assumption does not affect the outcome of this study.

According to the defined mathematical model, the software was developed to analyze the tribological parameters of an excavating manipulator in hydraulic excavators with continuous tracks on the basis of the measured quantities of the state of the excavator under exploitation conditions. The following quantities of the state of the excavator were measured: displacement of the support and movement mechanism, angle of the platform rotation, motion of hydraulic cylinders and pressures of the hydrostatic system in the ducts of the platform drive hydraulic motor, and the ducts of hydraulic cylinders in drive mechanisms of the excavator manipulator. By using the developed software, the analysis of the tribological parameters of drive mechanisms in a three-link manipulator of an excavator with continuous tracks of $17000 \mathrm{~kg}$ in mass and $70 \mathrm{~kW}$ in power was carried out when operating under exploitation conditions with an excavating bucket of $0.6 \mathrm{~m}^{3}$ in volume. 


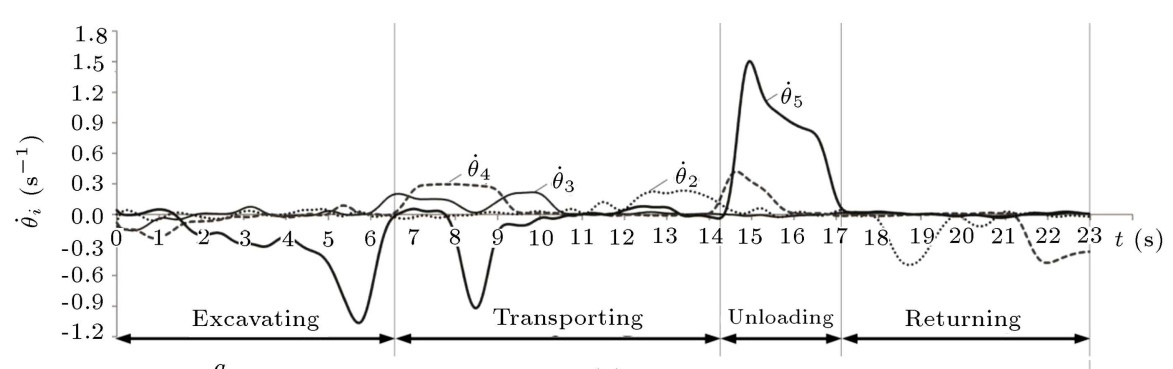

(a)

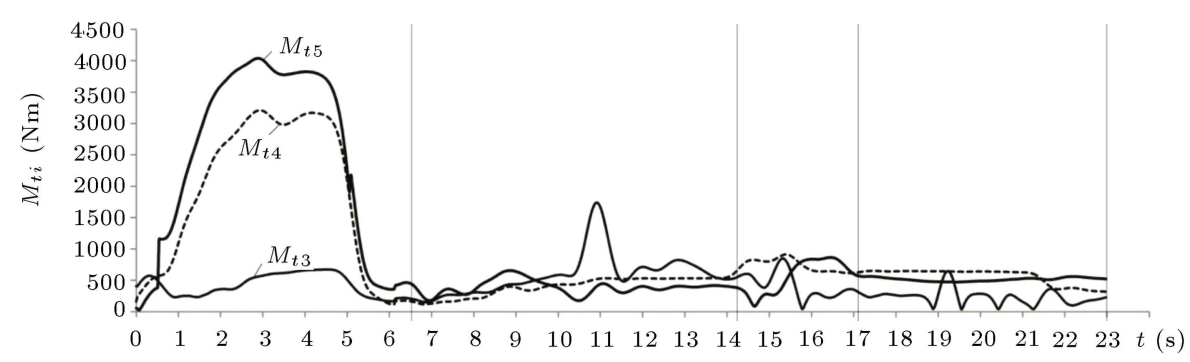

(b)

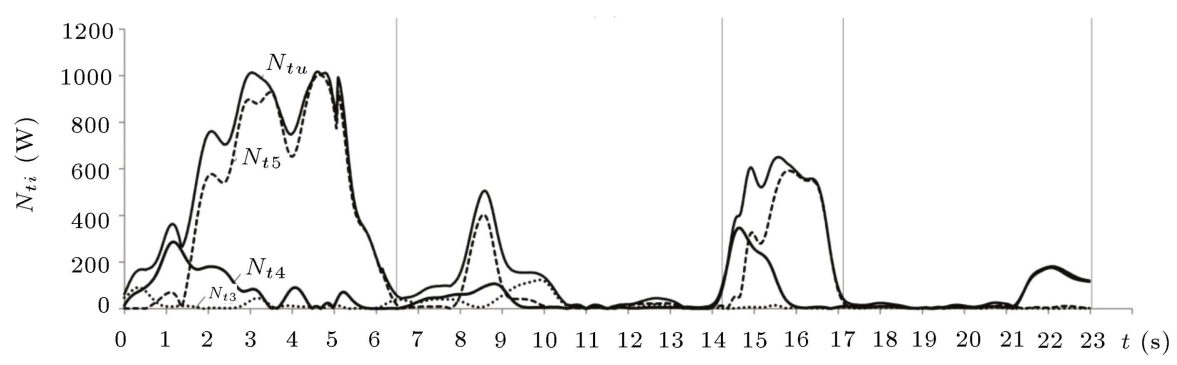

(c)

Figure 4. Experimental tribological analysis of the excavator: (a) Angular velocities $\dot{\theta}_{2}, \dot{\theta}_{3}, \dot{\theta}_{4}, \dot{\theta}_{5}$, (b) moments of friction $M_{t 3}, M_{t 4}, M_{t 5}$, and (c) loss of power $N_{t 3}, N_{t 4}, N_{t 3}, N_{t u}$ due to friction in joints $O_{3}, O_{4}$ and $O_{5}$ of the kinematic chain of the excavator.

Part of the research results is given graphically in a diagram that shows the change in angular velocities (Figure 4(a)), moments of friction (Figure 4(b)), and the loss of power due to friction (Figure $4(\mathrm{c})$ ) in the joints $O_{3}, O_{4}$, and $O_{5}$ of the manipulator kinematic chain. The obtained results show that the highest moments of friction and the greatest losses of power due to friction occur during the digging operation, at the beginning of the transfer operation, and finally during the operation of the unloading of the excavated material.

\section{Tribological optimization criterion}

By synthesizing drive mechanisms of hydraulic excavator manipulators, for the same given input hydrostatic power parameters, one can define several variant solutions of manipulator drive mechanisms with the same range of movement and drive moments and, yet, different parameters such as transformation and transmission. For example, the same range of movement angles $\theta_{i o}$ (Figure 5) and the same drive moment $M_{c i}$ of the executive mechanism link can be achieved using the same transformation parameter (the same size of the hydraulic cylinder), but with a different transmission parameter (Figure 5(a)-(c)), where, in principle, the executive mechanism link can be in the form of a one-armed (Figure 5(a) and (b)) or two-armed lever (Figure 5(a) and (b)). Furthermore, also possible are the variant solutions of drive mechanisms with lower transformation, but higher transmission parameters (Figure 5(c)), and vice versa (Figure 5(d)).

To choose an optimal solution for drive mechanisms in a hydraulic excavator manipulator among a set of possible variant solutions, basic aim functions are set: maximum performance and minimum excavator power loss. The tribological criterion concerned with determining optimal drive mechanism parameters for an excavator manipulator is defined to minimize the loss of power due to friction between the elements of mechanism joints:

$$
K_{t}=\min \left(\sum_{i}^{n} N_{t i}\right),
$$




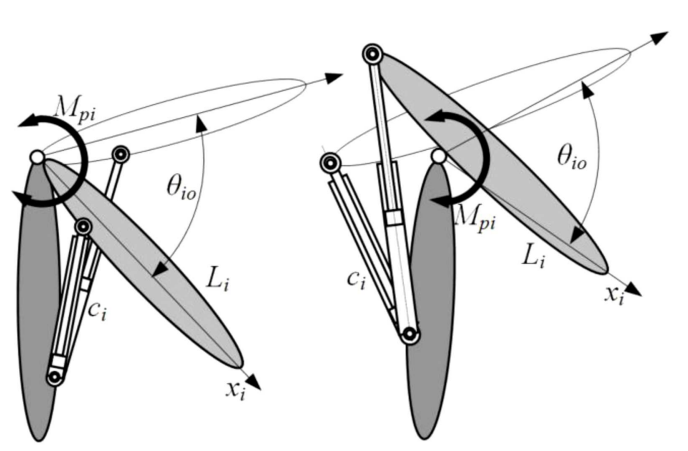

(a)

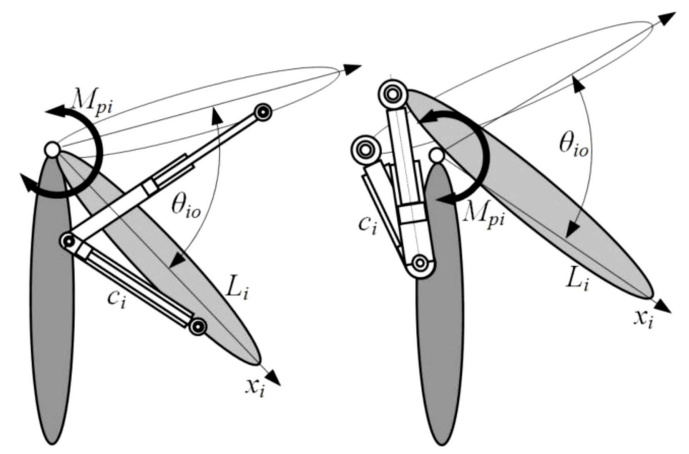

(c)

(d)

Figure 5. Possible variant solutions of manipulator drive mechanisms.

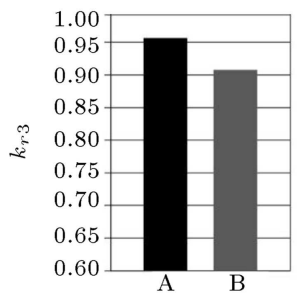

(a)

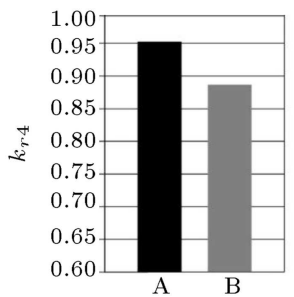

(b)

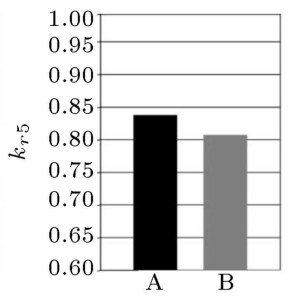

(c)

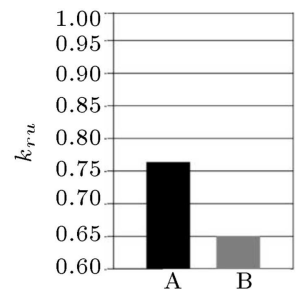

(d)

Figure 6. Indicators of the tribological optimization criterion $k_{r}$ for variant solutions of excavators A and B: (a) Boom mechanism $k_{r 3}$, (b) stick mechanism $k_{r 4}$, (c) bucket mechanism $k_{r 5}$, and (d) total manipulator indicators $k_{r u}$ of excavators $\mathrm{A}$ and B.

where $n$ is the number of manipulator drive mechanisms.

As a relative indicator $k_{r t}$ of the tribological optimization criterion, the mechanical efficiency of drive mechanisms is defined for a certain number of positions of the manipulator in the entire working range of the excavator:

$$
\begin{aligned}
k_{r t}= & \frac{1}{n_{3} n_{4} n_{5} n_{w}} \sum_{s=1}^{n_{3}} \sum_{r=1}^{n_{4}} \sum_{k=1}^{n_{5}} \sum_{w=1}^{n_{w}} \\
& \prod_{i=3}^{5} \frac{M_{i s r k w}}{\left(M_{i s r k w}+M_{t i \text { srkw }}\right)},
\end{aligned}
$$

where $M_{i \text { srkw }}$ is the loading moment of the manipulator drive mechanism without considering the friction resistive force of mechanism joints; $M_{t i}$ srkw is the moments of friction resistive force of the joints with indices that define the moments for a specific position of the boom $(s)$, stick $(r)$, and bucket $(k)$ and the specific acting direction of the digging resistive force $(w) ; n_{3}$ is the number of boom positions in its movement range; $n_{4}$ is the number of stick positions in its movement range for a given boom position; $n_{5}$ is the number of bucket positions in its movement range for a given stick position; and $n_{w}$ is the number of directions of digging resistive force.

By optimizing excavator drive mechanisms, a subprogram was developed to determine the indicators of the tribological optimization criterion. At the input of the subprogram, the following are set: data files with parameters of the excavator kinematic chain links, data files with variant solutions of the manipulator drive mechanisms, the desired number of manipulator positions in the entire working range of the excavator, surface pressure and coefficient of friction between the elements of manipulator joints.

The subprogram first discovers the possible digging resistive force and the corresponding forces in the manipulator joints for a specific position of the excavator kinematic chain and a specific acting direction of the digging resistive force. According to the highest values of forces of joints and mechanical characteristics of joint elements, the diameters of shafts (clevis pins) of joints are determined. After that, the subprogram determines the necessary drive moments of drive mechanisms without the influence of friction in joints and the moments of the resistive force of friction between the relatively mobile joint elements. Finally, the relative indicator of the tribological optimization criterion is found for the entire range of excavators.

For example, the relative tribological optimization indicators were determined for two variant solutions of excavators A and B using the developed subprogram (Figure 6). The obtained results showed that the drive mechanisms with lower transformation and higher transmission parameters had greater values of indicators of the tribological optimization criterion. 


\section{Conclusion}

The conducted tribological analyses and obtained results showed that the synthesis of manipulator drive mechanisms of hydraulic excavators should strive for lower transformation and higher transmission mechanism parameters as far as the spatial limitations of the installation of hydraulic cylinders are concerned. For the same power parameters of the excavator hydrostatic system, drive mechanisms of an excavator manipulator with lower transformation and higher transmission parameters of drive mechanisms experienced lower loads on joints for the same external mechanism loads. Smaller loads on mechanism joints lead to lower friction resistive force, less power loss, and lower wear between the joint elements, which increase the total mechanical efficiency and lifecycle of the excavator drive mechanisms. The defined tribological optimization criterion of manipulator drive mechanisms of hydraulic excavators represents the loss of power of the machine driving mechanism due to friction between joint elements of manipulator drive mechanisms. The criterion has a general character and can be used for the synthesis of lever mechanisms of manipulators and other mobile machines.

\section{Acknowledgement}

The paper presents a part of the research conducted within the technological development project no. 035049 which is funded by the Ministry of Education and Science of the Republic of Serbia.

\section{References}

1. Qiu, Q., Li, B., and Feng, P. "Optimal design of hydraulic excavator working device based on multiple surrogate models", Advances in $\mathrm{Me}$ chanical Engineering, 8(5), pp. 1-12 (2016). DOI: $10.1177 / 1687814016647947$

2. Pang, X., Gu, X., Chen, J., and Wang, Y. "Structural analysis and optimized design of working device for backhoe hydraulic excavator", 14th IFToMM World Congres, Taipei, Taiwan, pp. 588-594 (2015).

3. Li, X., Wang, G., Miao, S., and Li, X. "Optimal design of a hydraulic excavator working device based on parallel particle swarm optimization", J. Braz. Soc. Mech. Sci. Eng., 39(10), pp. 3793-3805 (2017). DOI: $10.1007 / \mathrm{s} 40430-017-0798-5$

4. Yin, G., Lin, S., and Perlurst, D.B. "Multidisciplinary design and collaborative optimization for excavator backhoe device", J. of Eng. Sci. and Technol. Rev., 7(2), pp. 99-105 (2014).

5. Chen, J., Qing, F., and Pang, X.J. "Mechanism optimal design of backhoe hydraulic excavator working device based on digging paths", J. of Mech.
Sci. and Technol, 28(1), pp. 213-222 (2014). DOI: 10.1007/s12206-013-0955-4

6. Janošević, D., Pavlović, J., Jovanović, V., and Petrović, P. "A numerical and experimental analysis of the dynamic stability of hydraulic excavators", Facta Universitatis Series: Mechanical Engineering, 16(2), pp. 157-170 (2018). DOI: 10.22190/FUME180404015J

7. Jovanović, V., Janošević, D., Pavlović, J. "Analysis of the influence of the digging position on the loading of the axial bearing of slewing platform drive mechanisms in hydraulic excavators", Facta Universitatis Series: Mechanical Engineering, (accepted for publication) (2019). DOI: 10.22190/FUME190225020J

8. Chackoa, V. and Khan, Z.A. "Dynamic simulation of a mobile manipulator with joint friction", Tribology in Industry, 39(2), pp. 152-167 (2017). DOI: 10.24874/ti.2017.39.02.02

9. Turis, J., Beňo, P., and Bialy, W. "The optimal tribotechnical factors in the design of machines - environmental element in the production systems", Management Systems in Production Engineering, 26(4), pp. 207-211 (2018). DOI: 10.1515/mspe-2018-0033

10. Janošević, D., Jovanović, V., and Petrović, N. "Spectra of slewing bearing load of a rotating platform drive in hydraulic excavators", Scientia Iranica, Transactions on Mechanical Engineering (B), 22(3), pp. 825-834 (2015).

11. Mitrev, R., Janošević, D., and Marinković, D. "Dynamical modelling of hydraulic excavator considered as a multibody system", J. Techn. Gazette, 24(2). pp. 327-338 (2017). DOI: 10.17559/TV-20151215150306

12. Mitrev, R., Tudjarov, B., and Todorov, T. "A cloudbased expert system for synthesis and evolutionary optimization of planar linkages", Facta Universitatis Series: Mechanical Engineering, 16(2), pp. 139-155 (2018). DOI: 10.22190/FUME180420016M

13. Khan, Z.A., Chacko, V., and Nazir, H. "A review of friction models in interacting joints for durability design", Friction, 5(1), pp. 1-22 (2017). DOI: 10.1007/s40544-017-0143-0

14. Bojić, N., Milčić, D., Banić, M., Mijajlović, M., and Nikolić, R. "Radial force impact on the friction coefficient and temperature of a self-lubricating plain bearing", Facta Universitatis Series: Mechanical Engineering, 15(3), pp. 427-437 (2017). DOI: 10.22190/FUME160830010B

\section{Biographies}

Jovan Pavlović received her MSc degree from University of Niš, Serbia, Mechanical Engineering Faculty. He pursued his studies $\mathrm{PhD}$ degree in Mechanical Engineering. His research interests include mechanical engineering and transport technology with focus on the earthmoving machinery.

Dragoslav Janošević is a Professor at the Mechanical Engineering Faculty at University of Nis, Serbia. He 
worked as a Mechanical Engineer in the company IMK 14 Research \& Development in Heavy Machinery, Krusevac, Serbia. He received his $\mathrm{PhD}$ in Mechanical Engineering Faculty in 1997. His research interests include transport technology and logistics.
Vesna Jovanović is an Assistant Professor and received her $\mathrm{PhD}$ degree from University of Nis, Serbia, Mechanical Engineering Faculty. Her research interests include mechanical engineering and transport technology with focus on the earthmoving machinery. 